\title{
Los experimentos mentales como género literario en el debate sobre identidad personal
}

\author{
Thought Experiments as Literature in the Debate on Personal \\ Identity
}

ALFONSO MUÑOZ CORCUERA*

\begin{abstract}
Resumen: El uso de experimentos mentales tradicionalmente ha sido uno de los principales recursos metodológicos en el debate sobre la identidad personal. Sin embargo, en las últimas décadas han recibido un creciente número de críticas. Esto ha provocado que muchos filósofos interesados en el problema de la identidad personal los hayan abandonado. Es el caso de la mayoría de los teóricos de la identidad narrativa. En este artículo comparo las críticas de Wilkes a los experimentos mentales con las de Ricoeur. Al hacerlo, espero demostrar que sus críticas se cancelan mutuamente, al menos de manera parcial, y que los teóricos de la identidad narrativa están comprometidos a aceptar el uso de experimentos mentales.
\end{abstract}

Palabras clave: Identidad personal, Experimentos mentales, Wilkes, Ricoeur, Narrativismo.

\begin{abstract}
The use of thought experiments has been one of the main methodological resources in the debate on personal identity. However, over the past decades, there has been an increasing amount of criticism against them. This has caused that many philosophers interested in the problem of personal identity have abandoned this methodology. This is the case with most of narrative identity theorists. In the present paper, I compare Wilkes' criticisms against thought experiments with Ricoeur's. In doing so, I hope to show that they partially cancel each other, and that narrative identity theorists are committed to accept the use of thought experiments.
\end{abstract}

Keywords: Personal Identity, Thought Experiments, Wilkes, Ricoeur, Narrativism.

\section{Introducción}

El uso de experimentos mentales ha sido tradicionalmente uno de los recursos metodológicos preferidos por los filósofos interesados en el problema de la identidad personal. Desde el cambio de cuerpos entre un príncipe y un zapatero propuesto por Locke en el siglo XVII hasta la actualidad han sido innumerables los distintos experimentos mentales que los filósofos han utilizado para poner en marcha su pensamiento: duplicaciones de personas,

Recibido: 30/05/2017. Aceptado: 20/01/2018.

* Investigador posdoctoral, Facultad de Filosofía, Universidad Complutense de Madrid. Correo electrónico: alfonso.m.corcuera@gmail.com. Líneas de investigación: Identidad personal en el terreno de la filosofía analítica. Para la escritura de este trabajo conté con una beca posdoctoral del programa de becas para estancias posdoctorales de la Universidad Nacional Autónoma de México. Publicaciones recientes: (2016) "Understanding Real and Fictional Persons: Narrative Negotiations Seen Through Cognitive Poetics", Philosophical Papers, 45 (1-2), 241-265; (2016). "Persons, Characters and the Meaning of 'Narrative"”, en R. Winkler (Ed.), Identity and Difference: Contemporary Debates on the Self (pp. 37-61), London, Palgrave Macmillan. 
fusiones, teletransportaciones, trasplantes cerebrales (véanse por ejemplo Parfit, 1984; Shoemaker, 1964; Williams, 1957, 1970)...

Sin embargo, según los experimentos se volvían cada vez más bizarros -por ejemplo piénsese en el experimento que Parfit bautizó como "Mi división", que incluye a tres trillizos y un doble de trasplante de los hemisferios cerebrales de uno de los trillizos a sus dos hermanos (Parfit, 1984, pp. 254-255)-, su uso empezó a ser cada vez más cuestionado por distintos filósofos, que bien postulaban su completo abandono o al menos su uso con serias limitaciones (vid. Gendler, 2002; Quine, 1972; Rovane, 1998; Sorensen, 1999; van Inwagen, 1997; Wilkes, 1988). De hecho, si uno compara la situación actual con la vivida antes de los años ochenta del siglo pasado, resulta llamativa la disminución en el uso de experimentos mentales. No en vano las teorías de la identidad narrativa, una de las posiciones más fuertes en el debate actual, apenas hacen uso de ellos. Por poner algunos ejemplos, Dennett y Schechtman, aunque no los rechazan por completo sí expresan sus reparos. Dennett piensa que un experimento que simplifique demasiado un problema puede generar más confusión que ayuda (Dennett, 2013, p. 321). Schechtman por su parte opina que si un experimento plantea una situación muy alejada de nuestro mundo directamente impide que podamos aplicar nuestros conceptos para comprenderla (Schechtman, 1996, pp. 133-134). Pero quizá el ejemplo paradigmático de la postura de las teorías narrativas sobre este aspecto sea la de Ricoeur, quien critica duramente el uso que Parfit y otros teóricos analíticos habían hecho de los experimentos mentales y propone su completa sustitución por las comparaciones entre lo que somos y los personajes literarios (vid. Ricoeur, 1990).

La crítica de Ricoeur al uso de los experimentos mentales contrasta con la de Wilkes, posiblemente la crítica más importante al respecto. Wilkes también abogó por el abandono del uso de experimentos mentales en la investigación sobre identidad personal. Pero al contrario que Ricoeur, lo hizo precisamente porque consideraba que se parecían demasiado a la literatura y que por tanto no podían tener ningún interés para la investigación filosófica. De este modo, aunque ambos autores coinciden en la importancia de abandonar el uso de experimentos mentales, sus propuestas van en sentidos contrarios. Para Ricoeur, los experimentos mentales son demasiado científicos -o cientificistas- y se deben sustituir por ejemplos literarios. Para Wilkes, son demasiado literarios y se deben sustituir por experimentos científicos reales.

Esta divergencia entre las dos críticas creo que sitúa a las teorías narrativas de la identidad personal en una posición privilegiada para abogar por el uso de ambas metodologías. Considero que el análisis de Wilkes es correcto, y los experimentos mentales al menos comparten con la literatura una serie de rasgos importantes. Sin embargo, su propuesta de abandono creo que no está motivada. Aunque los experimentos mentales en la investigación sobre la identidad personal no puedan adaptarse al esquema de los experimentos científicos, esto no es algo malo. Ricoeur y el resto de los partidarios de las teorías narrativas están en lo cierto al defender que las comparaciones entre lo que somos y los personajes literarios puede tener un resultado productivo. Siendo así, aunque quizá otras posturas dentro del debate de la identidad personal deban renunciar al uso de experimentos mentales ( $c f r$. Beck, 2006; Coleman, 2000), los partidarios de las teorías narrativas deberían incorporarlos a su repertorio.

El esquema de mi argumentación será el siguiente: En primer lugar explicaré el concepto de "experimento mental" y haré un repaso de las críticas a su uso que hacen Wilkes y otros 
autores coincidentes con ella. Tras ello presentaré brevemente la postura de Ricoeur al respecto. Finalmente, abogaré (1) por una interpretación de los experimentos mentales más laxa que la de Wilkes que permita su asimilación a la literatura y (2) por una concepción más amplia de la literatura que la de Ricoeur que no excluya por principio los experimentos mentales. Bajo esta nueva perspectiva, considero que los teóricos de la identidad narrativa estarían en disposición de recurrir a ambas metodologías. ${ }^{1}$

\section{Los experimentos mentales según Wilkes}

De acuerdo con Wilkes, un experimento mental es una actividad consistente en imaginar un mundo posible en el que un determinado conjunto de estados se da y tratar de extraer conclusiones acerca de lo que diríamos si esa situación ocurriese realmente. Esto es, si nosotros habitásemos en ese mundo posible. Para que las conclusiones puedan aplicarse después con más facilidad al mundo real, el mundo posible del experimento debe ser igual al nuestro en todos los aspectos relevantes excepto en los postulados por el experimento (Wilkes, 1988, p. 2). Esta definición trata de ser lo más abarcadora posible, de tal modo que incluya tanto los experimentos mentales que se utilizan en las ciencias naturales como los que se utilizan en filosofía.

Dado que los experimentos mentales son un recurso de investigación que utilizan tanto los científicos naturales como los filósofos, Wilkes y Sorensen consideran que para poder extraer conclusiones fiables los experimentos mentales deben construirse siguiendo el modelo de los experimentos científicos reales y estar sujetos a las mismas restricciones (Sorensen, 1999, p. 3; Wilkes, 1988, pp. 6-7). En concreto, deben llevarse a cabo con un objetivo definido, aislar la variable cuya influencia sobre un determinado problema quiera estudiarse y fijar el trasfondo de tal modo que al permanecer estable no interfiera en los resultados del experimento (Wilkes, 1988, p. 7).

Uno de los principales motivos para utilizar un experimento mental es que, por algún motivo, dicho experimento sea imposible de llevarse a cabo en la realidad. Dicha imposibilidad puede ser evadida presuponiendo que existe un mundo posible en el que no es imposible hacerlo. Sin embargo es importante tener en cuenta si evitar dicha imposibilidad tiene alguna relevancia para las conclusiones del experimento, de tal modo que ignorarla haría que las conclusiones no fuesen aplicables a nuestro mundo. Por ejemplo, presuponer que un ser humano podría viajar a la velocidad de la luz es aceptable si nuestro objetivo es investigar sobre un problema de la física de partículas. Pero si nuestro objetivo es investigar sobre las condiciones de supervivencia de los seres humanos, nuestra presuposición inva-

1 Es importante señalar que el presente artículo da por supuesto que las comparaciones entre lo que somos y las ficciones literarias pueden ser provechosas. Esta presuposición, compartida en términos generales por todos los partidarios de las teorías narrativas de la identidad (vid e.g. Dennett, 1992; MacIntyre, 2007; Schechtman, 1996; Taylor, 2001), puede ser atacada desde dos frentes. Por un lado, puede argumentarse que la ficción no tiene ningún valor cognitivo y que por tanto no pueden extraerse conclusiones de ella. Por otro, aunque se conceda que la ficción puede tener un valor cognitivo, puede aducirse que no existe ninguna similitud entre lo que somos y los personajes literarios, por lo que las comparaciones son engañosas (vid. Lamarque, 2007). En cuanto a la primera crítica, Zarnitsyn (2013) realiza una defensa contundente. En cuanto a la segunda, véase mi respuesta (Muñoz-Corcuera, 2016). 
lidará los resultados que queramos extrapolar. En este sentido, los filósofos interesados en el problema de la identidad personal únicamente ponen una restricción a sus experimentos mentales: que sean lógicamente posibles. Esto es, que no contengan ninguna contradicción lógica. Wilkes considera que esta restricción es demasiado débil y considera que en su lugar los experimentos mentales deben construirse recurriendo a otro tipo de posibilidad: la teórica. Esto es, que no entren en contradicción con ninguna de nuestras teorías científicas (Wilkes, 1988, p. 18). En cualquier caso regresaré sobre esto más adelante.

Una vez aclarado qué se entiende por "experimento mental”, podemos pasar a las críticas que Wilkes realiza contra su uso en el ámbito de la investigación sobre identidad personal. En concreto me gustaría señalar tres críticas que, a juicio de Wilkes, provocan que estos experimentos mentales no puedan adaptarse al modelo de los experimentos científicos reales y que por tanto deban ser considerados como análogos a la literatura. La primera se refiere a la falta de determinación del trasfondo relevante para la interpretación de los experimentos mentales que se utilizan en identidad personal. La segunda señala que el hecho de que "persona" sea un término de sentido común provoca incertidumbre en cuanto a su aplicabilidad en contextos imaginarios. Finalmente, la tercera se dirige al tipo de "posibilidad" que debe ser relevante para que un experimento mental sea valioso.

Evidentemente, estas tres críticas se encuentran relacionadas entre sí, de tal modo que la división es hasta cierto punto artificial. Por poner un ejemplo, la crítica al tipo de "posibilidad" relevante tiene relación con el hecho de que "persona" es un término de sentido común sobre el que no se puede estar seguro de sus implicaciones lógicas. Del mismo modo, el hecho de que "persona" sea un término de sentido común hace que la determinación del trasfondo relevante para el experimento sea más complicado que en el caso de experimentos que implican términos referidos a conceptos científicos o a clases naturales. En cualquier caso creo que la división será útil por motivos didácticos.

\subsection{La indeterminación del trasfondo}

El primer tipo de crítica que me gustaría señalar es el referido a la importancia de que el trasfondo que rodea a la situación objeto del experimento mental se encuentre bien determinado. Como señalaba en $\S 2$, de acuerdo con Wilkes y Sorensen un experimento mental es un caso límite de un experimento real y debe estar sometido a las mismas restricciones que estos para ofrecer resultados interesantes. Especialmente debe aislar la variable cuya influencia se va a estudiar y determinar el trasfondo para asegurarse de que ningún factor relevante influya en el resultado. Por poner un ejemplo, si se quiere comprobar el papel de la continuidad psicológica en el problema de la identidad personal a través del tiempo, la situación que plantee el experimento mental debe aislar esa variable del trasfondo, de tal forma que el resto de factores que puedan afectar al problema permanezcan tal y como son en nuestro mundo. De este modo, si en el experimento se introduce un cambio que afecta únicamente a la continuidad psicológica y como resultado se produce un cambio en nuestros juicios sobre la identidad personal a través del tiempo, quedará perfectamente claro que el resultado se debe exclusivamente al cambio que afecta a la continuidad psicológica. Sin embargo, si no se determina el trasfondo que rodea al experimento, no se podrá aislar correctamente la variable a estudiar y no quedará claro si los cambios en el resultado del 
experimento se deben a los cambios en la variable o a cambios en el trasfondo que no se han tenido en cuenta. Volviendo sobre el ejemplo, si el experimento no especifica cómo es la sociedad en la que se desarrolla la situación, puede ser que los cambios en nuestros juicios sobre la identidad a través del tiempo no se deban al cambio que afecta a la continuidad psicológica, sino a un cambio en la estructura social que hace que el concepto de "persona" se aplique de un modo distinto en ese mundo posible (Wilkes, 1988, p. 11).

Invariablemente, según Wilkes, ninguno de los experimentos mentales utilizados por los filósofos interesados en el problema de la identidad personal determinan de modo adecuado el trasfondo. Situación que, además, se ve agravada por el hecho de que "persona" es un término de sentido común y por tanto sus implicaciones tampoco están bien definidas (véase §2.2). Siendo así, es comprensible que distintos lectores de un mismo experimento mental lleguen a conclusiones opuestas: dado que el trasfondo no está determinado por el experimento, cada lector lo determina del modo que considera apropiado tal y como sucede en la literatura (Wilkes, 1988, pp. 8-12). ${ }^{2}$ En consecuencia, en los experimentos mentales utilizados en la investigación en identidad personal nunca es posible saber si los resultados son debidos a los cambios introducidos explícitamente por el experimento o al modo en que el lector determinó el trasfondo.

\subsection{El uso de términos de sentido común}

El segundo tipo de crítica se refiere al hecho de que el término "persona" es un término de sentido común y por tanto no es un buen candidato para ser utilizado en un experimento mental. En este sentido, Wilkes argumenta que existe una diferencia importante entre los experimentos mentales utilizados en las ciencias naturales y los utilizados en la filosofía, pues la ciencia se ocupa de explorar términos referidos a clases naturales -no entraremos aquí a discutir sobre el concepto de "clase natural"- mientras que la filosofía se ocupa mayoritariamente de términos de sentido común. La ciencia define los términos referidos a clases naturales con precisión, establece con claridad sus implicaciones y formula leyes al respecto. Por el contrario, los términos de sentido común están definidos vagamente y poseen multitud de implicaciones que dependen de factores contextuales difíciles de determinar (Wilkes, 1988, pp. 13-14). Siendo así, en los experimentos mentales que involucran a clases naturales es fácil aislar la variable que se quiere estudiar, determinar qué factores contextuales son relevantes y cuáles pueden afectar a la validez del experimento. Por el contrario, en los experimentos utilizados en la investigación sobre identidad personal, es casi

2 El hecho de que las respuestas a un determinado experimento mental puedan variar de una persona a otra es un problema para esta metodología que ha sido señalado por diversos autores (vid. e.g. Johnston, 1987; Rovane, 1998, pp. 35-64; Williams, 1970). Si distintos lectores de un experimento dan respuestas distintas frente al mismo, entonces el experimento no es capaz de demostrar nada, sino que sólo genera confusión. De hecho, de acuerdo con Beck, uno de los escasos defensores contemporáneos del uso de experimentos mentales, ésta podría ser su única limitación: si estadísticamente no existe una respuesta mayoritaria frente a un cierto experimento, entonces no puede extraerse conclusión alguna del mismo. Sin embargo, de acuerdo con Beck, éste no es el caso de los experimentos mentales que se usan en el debate sobre la identidad personal (Beck, 2006, pp. 50-52). Romerales parece coincidir con Beck en este punto y en su rápido análisis de los problemas que afectan a los experimentos mentales señala la variabilidad de nuestras intuiciones como la única de sus limitaciones que no admite respuesta (Romerales , 1993, pp. 110-1). 
imposible aislar una variable en concreto y determinar qué factores se verán afectados por la situación propuesta, de tal modo que, incluso si el experimento produce algún resultado, no se puede saber si las conclusiones son extrapolables al mundo real.

\subsection{La posibilidad de concebir y el tipo de posibilidad relevante}

Finalmente, el tercer tipo de crítica se refiere al concepto de "posibilidad" y tiene dos vertientes. Por un lado se dirige a la confusión existente entre que algo sea concebible y el que ese algo sea lógicamente posible. Por otro lado, al hecho de que la posibilidad lógica exigida por los teóricos de la identidad personal para la validez de sus experimentos mentales quizá no es lo suficientemente estricta como para obtener resultados confiables.

En cuanto a la primera vertiente, los críticos de los experimentos mentales señalan que a menudo no se distingue entre el hecho de que sea posible concebir una situación y el hecho de que dicha situación sea lógicamente posible. Ni todo lo concebible es lógicamente posible, ni todo lo lógicamente posible es concebible. Por poner un ejemplo, no podemos concebir cómo es sentir los gradientes de presión del agua. Sin embargo los peces lo hacen, por lo que se trata de una posibilidad que de hecho se da en el mundo real (Sorensen, 1999, p. 37). Del mismo modo, podemos concebir una barra de hierro que flote en el agua. Sin embargo, para flotar en el agua un objeto debe tener una gravedad específica menor a 1 y el hierro tiene una gravedad específica que oscila entre los 7,4 y los 7,8. Siendo así, aunque es concebible una barra de hierro que flote en el agua, se trata de una situación que implica una imposibilidad lógica, pues un objeto no puede tener al mismo tiempo una gravedad específica mayor y menor que 1 (Wilkes, 1988, p. 18).

Lo que podemos concebir está determinado por nuestra biología, nuestro trasfondo cultural, nuestras creencias y conocimientos individuales, por el modo en que se nos plantee una determinada situación e incluso por el orden en que se nos presente una serie de situaciones (Johnston, 1987, pp. 66-67; Rorty, 1980; Sorensen, 1999, pp. 27-29; Wilkes, 1988, pp. 15-17; Williams, 1970). Ser capaz de concebir una situación no tiene nada que ver con el hecho de que sea lógicamente posible. En consecuencia, se deben repensar los experimentos mentales utilizados por los teóricos de la identidad personal, pues quizá no sólo sean físicamente irrealizables por una mera imposibilidad técnica, sino que a pesar de ser concebibles también oculten en su seno una imposibilidad lógica.

En este sentido, recurrir como única restricción para la validez de un experimento mental al concepto de "posibilidad lógica" es problemático. Debemos tener en cuenta que si recurrimos directamente a este tipo de posibilidad para plantear un experimento es necesario asegurarse primero, tal y como señalábamos en \$2.1, de que la descripción del mismo sea la suficientemente clara e incluya todos los factores relevantes (Wilkes, 1988, p. 43). De otro modo podríamos estar planteando una situación concebible sin contradicciones aparentes pero que en realidad contiene una imposibilidad lógica, como sucede en el caso de la barra de hierro que flota en el agua. Por poner algunos ejemplos, los experimentos mentales utilizados en la investigación sobre identidad personal que involucran una fusión o una fisión de personas, el trasplante de un único hemisferio cerebral o el "copiado" de las conexiones neuronales de un cerebro a otro, son incompatibles con diversos hechos biológicos que no están lo suficientemente especificados en los experimentos pero que son esenciales para 
considerar que seguimos siendo seres humanos (van Inwagen, 1997, pp. 305-308; Wilkes, 1988, pp. 36-37).

Podría alegarse que este tipo de experimentos mentales se refieren a mundos posibles de tipo fantástico en los que el hecho biológico que genera la imposibilidad no existe. Sin embargo, debemos considerar que los hechos biológicos no se dan de forma aislada, sino que unos dependen de otros (Wilkes, 1988, p. 31). Eliminar un hecho biológico en el mundo del experimento implicaría modificar también otros hechos que a su vez implicarían modificaciones ulteriores. E incluso si presuponemos que efectivamente puede construirse un mundo lógicamente posible que incluya todas las modificaciones necesarias para que el experimento tuviese lugar, nos encontraríamos con que se trataría de un mundo diferente del nuestro en un grado indeterminado. Y no es posible saber hasta qué punto las conclusiones que se puedan extraer acerca de lo que podría suceder en un mundo así serían aplicables a nuestro mundo. En palabras de Wilkes:

In sum, we cannot extract philosophically interesting conclusions from fantastical thought experiments. We cannot do this because we have the following choice: either (a) we picture them against the world as we know it, or $(b)$ we picture them against some quite different background. If we choose the first, then we picture them against a background that deems them impossible - that insists that hemisphere transplants (for instance) violate fundamental biological and physical laws. If we choose $(b)$, then we have the realm of fantasy, and fantasy is fine to read; but it does not allow for philosophical conclusions to be drawn, because in a world indeterminately different we do not know what we would want to say about anything. (Wilkes, 1988, p. 46)

Esta referencia de Wilkes a la fantasía señala otro de los problemas que tiene el recurrir como única restricción para la validez de un experimento mental al concepto de posibilidad lógica: los mundos posibles postulados por la literatura, incluso los propios de la literatura fantástica como la de Lewis Carroll o J. R. R. Tolkien, también están restringidos, según Wilkes (véase §5), por la posibilidad lógica (Wilkes, 1988, pp. 10, 17). Siendo así, los filósofos interesados en la identidad personal no tendrían ningún motivo para preferir que sus experimentos tuviesen una inspiración científica, sino que podrían recurrir igualmente a la literatura fantástica. De hecho, no hacerlo se puede considerar casi como un acto de mala fe, pues genera la ilusión de que sus experimentos mentales tienen una validez y un respaldo científico que en realidad no tienen. Si su única restricción es hablar de lo lógicamente posible, su propio trabajo como filósofos no se diferenciaría del de un escritor, pues al escribir experimentos mentales en realidad estarían escribiendo literatura (Wilkes, 1988, pp. 36, 44). Sin embargo, dado que la literatura plantea mundos en los que nuestros conocimientos científicos no tienen aplicación, es un disparate recurrir a ellos para saber cómo es nuestro mundo real regido por leyes científicas. Para Wilkes, los únicos experimentos mentales fiables, como decíamos anteriormente, son aquellos restringidos por la posibilidad teórica. En cualquier caso, ante la duda, la mejor alternativa para los teóricos de la identidad personal, de acuerdo con Wilkes, es abandonar los experimentos mentales por completo y sustituirlos por experimentos científicos reales que, siendo igual de desafiantes, no presentan ninguno de los problemas señalados. 


\section{Los experimentos mentales según Ricoeur}

Como señalaba en la introducción, pese a que Ricoeur rechaza también el uso de experimentos mentales en la investigación sobre la identidad personal, sus motivos son radicalmente opuestos a los de Wilkes. Si para Wilkes el hecho de que los experimentos mentales no se adapten al modelo que ofrecen los experimentos científicos los equipara a la literatura, para Ricoeur el problema es justamente el contrario: los experimentos mentales tienen un espíritu cientificista que impide una correcta comprensión del problema de la identidad personal. En su lugar habría que sustituirlos por las comparaciones entre lo que somos y los personajes literarios, pues la literatura tiene una concepción de lo que somos mucho más adecuada.

De acuerdo con Ricoeur, los experimentos mentales utilizados por los filósofos analíticos interesados en el problema de la identidad personal parten de un error de base: consideran el problema de la identidad exclusivamente en términos de mismidad (mêmeté en francés o sameness en inglés) e ignoran todo lo relativo a la ipseidad (ipséité o selfhood). Esta distinción entre mismidad e ipseidad puede ser entendida a la luz de las preguntas a las que responde cada tipo de identidad. Según Ricoeur, la identidad entendida en términos de mismidad es una respuesta a la pregunta “¿qué?”, mientras que la ipseidad tiene que ver con la pregunta “¿quién?”. Es decir, mientras que la mismidad tiene que ver con la dimensión objetiva, públicamente observable y analizable de la identidad, la ipseidad se refiere a la dimensión subjetiva de la misma. En términos analíticos podríamos decir que la ipseidad se refiere a la dimensión fenomenológica de la identidad, a lo que recientemente ha venido a denominarse "sense of mineness". En este sentido, señala Ricoeur, no es extraño que los experimentos mentales utilizados por los filósofos analíticos se centren en la manipulación del cerebro, "considerado como el equivalente de la persona" (Ricoeur, 1990, p. 162, trad. en 1996, p. 133). Pues a pesar de ser éste la base de nuestra vida mental, el cerebro difiere de otras partes de nuestro cuerpo en que "está desprovisto de cualquier estatuto fenomenológico y, por tanto, del rasgo de pertenencia mía” (Ricoeur, 1990, p. 159, trad. en 1996, p. 130).

$\mathrm{Al}$ entender la identidad exclusivamente en términos de mismidad, cuando los filósofos analíticos plantean por ejemplo un experimento que implica un cambio de cuerpo ignoran el hecho de que tenemos una experiencia fenomenológica de posesión sobre nuestro cuerpo, y que ésta se vería destruida con el cambio. Lo mismo sucede cuando plantean la posibilidad de borrar e implantar recuerdos en el cerebro de una persona, pues ignoran que los recuerdos deben ser experimentados como propios por su poseedor (Ricoeur, 1990, p. 163 , trad. en 1996, p. 134). En términos más generales, aunque los experimentos mentales propuestos por los filósofos analíticos presentan situaciones lógicamente posibles, ignoran que la existencia terrestre del ser humano impone (o debe imponer) restricciones más fuertes sobre lo que es lícito imaginar (Ricoeur, 1990, p. 162, trad. en 1996, pp. 132-3). En palabras de Benito Vicente, aunque un experimento mental no sea lógicamente imposible, puede ser empíricamente imposible, lo cual es suficiente para dudar de su relevancia para la investigación sobre el problema de la identidad personal (Benito Vicente, 2003, pp. 80-1). En este sentido las críticas de Ricoeur y Benito Vicente coinciden con la de Wilkes en lo que al problema del recurso a la posibilidad lógica se refiere. Aunque, a diferencia de ella, Ricoeur y Benito 
Vicente parecen proponer como alternativa el marco más restrictivo de la posibilidad metafísica, y no el de la posibilidad teórica de la que hablaba Wilkes.

Frente a los experimentos mentales, que enfocan el problema de la identidad exclusivamente en términos de mismidad, Ricoeur propone como metodología la utilización de las comparaciones entre las personas y los personajes literarios. Pues al contrario de lo que sucede con la ficción tecnológica, los personajes de la literatura son humanos como nosotros, por lo que su existencia ficcional "sigue estando sometida también a la restricción de la condición corporal y terrestre del hombre" (Ricoeur, 1990, p. 178, trad. en 1996, p. 150). En otras palabras, la literatura no atiende sólo a la mismidad, sino que también, y especialmente, se centra en la ipseidad.

\section{Una alternativa conciliadora}

La divergencia de criterios entre Ricoeur y Wilkes en lo que al carácter literario de los experimentos mentales y su valor para la investigación en el problema de la identidad personal se refiere invita a pensar en que quizá exista un punto de encuentro entre ambas críticas, de tal modo que una anule parcialmente a la otra. A lo mejor Wilkes es demasiado radical en su condena a los experimentos que no se adaptan al modelo que proveen las ciencias naturales y Ricoeur demasiado exigente en su concepción de lo que es literatura. Si así fuese, aún podríamos ofrecer una salvación a los experimentos mentales.

Como decíamos, las críticas de Wilkes al uso de experimentos mentales en la investigación sobre identidad personal parten del supuesto de que un experimento mental es un caso límite de un experimento real y que por tanto debe formularse con las mismas restricciones (véase §2). Sin embargo, se trata de un supuesto demasiado exigente. Sin duda algunos filósofos tratan de extraer conclusiones directamente de sus experimentos mentales y en ese sentido parecen querer amoldarse al modelo científico. Uno puede pensar por ejemplo en las conclusiones de Williams o de Shoemaker a partir de los experimentos de duplicación de personas (vid. Shoemaker, 1964; Williams, 1957). Sin embargo, considero que la principal motivación para el uso de experimentos mentales en la investigación sobre identidad personal, ya desde su inicio en el trabajo de Locke, no es tanto el utilizarlos como argumentos rigurosos de los que extraer conclusiones como el de poner a prueba nuestras intuiciones (vid. Locke, 1975). Lo que es más, al analizar nuestras intuiciones, los experimentos mentales no tienen como objetivo descubrir cómo es la realidad -como si tuviésemos un conocimiento innato sobre su estructura profunda-, sino conocer cuáles son nuestras creencias al respecto. Esto es, los experimentos mentales que se utilizan en la investigación sobre identidad personal no se dirigen al análisis de cómo son realmente las personas, sino al de cómo es nuestro concepto de "persona" (Beck, 2006, pp. 43-44). Son un tipo de experimento mental que Dennett denomina "bomba de intuición” (Dennett, 2013, pp. 5-6).

A diferencia de los experimentos mentales construidos de forma rigurosa siguiendo el modelo de los experimentos científicos reales, las bombas de intuición no tienen como objetivo el llegar a conclusiones definitivas, sino el servir de exploración para obtener una mejor comprensión del problema. En este sentido no presentan un escenario rígido, sino que tienen una función dialéctica sobre la que el filósofo regresa para modificar sus presupuestos originales, analizar nuevos argumentos y, en general, profundizar en su análisis (Diamond, 
2002, pp. 234-235). Desde esta perspectiva es interesante ver cómo Parfit, en referencia a las críticas sobre el sinsentido que supone preguntarse por nuestra supervivencia en un escenario radicalmente distinto al de nuestra situación real, señala que estas críticas podrían estar justificadas si, al enfrentarnos con esos experimentos mentales, no tuviésemos ninguna reacción. Sin embargo, este no es el caso. Las situaciones propuestas por los experimentos mentales movilizan con fuerza nuestras intuiciones acerca de lo que somos, y por tanto son un buen método para investigar cuáles son nuestras creencias al respecto (Parfit, 1984, p. 200). Nuestras creencias pueden estar erradas, desde luego. El supuesto de que nuestras creencias e intuiciones sobre nuestra supervivencia son relevantes para la investigación sobre el problema de la identidad personal descansa en las presuposiciones de que la identidad personal es lo que nos importa cuando nos preocupamos por nuestra supervivencia y que de algún modo nuestra preocupación egocéntrica, prudencial, por nuestro propio futuro es capaz de seguir o rastrear nuestra identidad metafísica. Estas presuposiciones pueden ser atacadas (vid.e.g. Beck, 2006; Martin, 1998). Pero en cualquier caso se trata de un problema distinto que no invalida el método de los experimentos mentales en sí, sino que en todo caso cuestiona la relevancia de sus resultados para el problema de la identidad personal.

Esta línea de respuesta es prometedora. Sin duda sería mentira decir que todos los experimentos mentales que se han utilizado en la investigación sobre el problema de la identidad personal siguen el modelo de las bombas de intuición, pues como Zarnitsyn señala, la línea que separa los experimentos mentales que tienen un carácter exploratorio de los que siguen el modelo de los experimentos científicos es difusa y a menudo ignorada por los filósofos (Zarnitsyn, 2013, p. 43). Además, las bombas de intuición también presentan sus propios problemas, pues, como Dennett señala, en ocasiones pueden ser más una fuente de confusiones que una ayuda (Dennett, 2013,p. 321). Especialmente cuando simplifican la situación en exceso e ignoran aspectos contextuales que podrían ser relevantes (véase §5). No obstante, la idea de considerar los experimentos mentales desde esta perspectiva abre una puerta a su uso que Wilkes consideraba cerrada.

Si aceptamos entonces que los experimentos mentales que utilizan los filósofos interesados en el problema de la identidad personal podrían ser útiles a pesar de ser un producto muy cercano a la literatura, ¿qué podemos decir entonces de la postura de Ricoeur? ¿Existe algún modo de compatibilizar su postura con la de Wilkes?

Como veíamos en $\$ 3$, en principio Ricoeur parece establecer una distinción clara entre los experimentos mentales y la literatura. Los primeros construyen mundos posibles dentro del marco de la posibilidad lógica y se dedican a explorar la identidad en términos de mismidad. Los segundos construyen mundos posibles dentro del marco de la posibilidad metafísica y exploran la identidad bajo su carácter de ipseidad. Sin embargo, si uno intenta profundizar en cómo Ricoeur entiende esta distinción, descubre que no sólo es problemática, sino que tampoco es tan tajante como podría parecer a primera vista.

En primer lugar, Ricoeur piensa que es imposible pensar la identidad exclusivamente en términos de mismidad o de ipseidad hasta sus últimas consecuencias (Ricoeur, 1990, pp. 146-147). La continuidad del carácter, ejemplo paradigmático del problema de la identidad personal entendido en términos de mismidad, no es otra cosa que una objetivización de algo radicalmente subjetivo. Nuestra vivencia personal, el modo en que nos hemos formado nuestra subjetividad, cristaliza y se sedimenta en una serie de rasgos estables que 
constituyen el carácter de una persona, gracias al cual es posible identificar a una persona de forma objetiva. Sin embargo, pese a tener una apariencia objetiva y ser por tanto propio de la mismidad, el carácter es en esencia algo subjetivo. Es un carácter que nos pertenece en un sentido íntimo, y cuya explicación de cómo lo hemos adquirido no puede hacerse al margen de la ipseidad (Ricoeur, 1990, p. 147).

Por otro lado, pensar la identidad exclusivamente en términos de ipseidad es también imposible. A partir del análisis de El hombre sin atributos de Musil, Ricoeur muestra cómo la literatura en ocasiones experimenta con personajes que dejan de tener un carácter estable y por tanto cuya identidad ya no puede entenderse en términos de mismidad. Sin embargo, al separar radicalmente la ipseidad de la mismidad, la propia identidad desaparece (Ricoeur, 1990, p. 177). Los personajes sin un carácter acaban siendo personajes sin identidad, que no son nada o que, en términos populares, sufren una crisis profunda de identidad al no poder entender (ni mantener) su propia continuidad a lo largo del tiempo (Ricoeur, 1990, p. 198).

Esta imposibilidad de pensar la mismidad y la ipseidad de forma separada se traduce en que ni los experimentos mentales afectan únicamente a la mismidad ni la literatura trata en exclusiva de la ipseidad. Los experimentos mentales que utilizan los filósofos analíticos se centran en la identidad entendida en términos de mismidad, pero no por ello dejan de afectar a la ipseidad. Cuando Parfit, gracias a sus experimentos mentales, llega a la conclusión de que la identidad no importa, es pertinente preguntarle a quién no le importa. La propia pregunta por la importancia de la identidad presupone un yo preocupado prudencialmente por sí mismo, lo cual es un elemento constitutivo de la ipseidad (Ricoeur, 1990, pp. 164-165). Por su parte el caso de El hombre sin atributos es un ejemplo extremo en el que una obra literaria se olvida por completo de la mismidad. Sin embargo podemos pensar en los cuentos de hadas como el ejemplo contrario, en el que los personajes tienen un carácter absolutamente estable que no cambia en absoluto a lo largo de toda la narración (Ricoeur, 1990, p. 176). En este sentido podemos ver que en realidad la diferencia entre los experimentos mentales y la literatura no es tan clara. Ambos tipos de ficciones pueden tratar la identidad tanto en términos de mismidad como de ipseidad.

Siendo así, de acuerdo con la postura de Ricoeur la única diferencia significativa entre los experimentos mentales y la literatura podría estribar en el tipo de posibilidad relevante para la construcción de sus mundos. Como decíamos, Ricoeur parece defender que mientras los experimentos mentales atienden exclusivamente a la posibilidad lógica, la literatura se rige por la más estricta posibilidad metafísica. Sin embargo esta posible diferenciación parece difícil de defender. ¿Son los mundos de los cuentos de hadas, a los que Ricoeur se refiere para hablar de personajes con un carácter estable, metafísicamente posibles? ¿O más bien, como señalaba Wilkes, los cuentos de hadas se rigen (véase §5) por la mera posibilidad lógica? Por otro lado, ¿no es la ciencia-ficción en sí misma un género literario? ¿Son entonces las obras de Philip K. Dick, en las que aparecen frecuentes manipulaciones sobre el cerebro análogas a las que aparecen en los experimentos mentales, metafísicamente posibles? Y si es así, ¿lo son por el mero hecho de que en las librerías se venden en la sección de "literatura"?

Teniendo en cuenta estas consideraciones, la crítica de Ricoeur parece sostenerse únicamente en base a un criterio temático. Las ficciones de inspiración tecnológica/futurista no son apropiadas para investigar sobre el problema de la identidad personal porque no prestan 
atención a la dimensión subjetiva de la existencia de las personas. Sin embargo esta crítica podría responderse del mismo modo en que respondimos a Wilkes. Quizá los experimentos mentales no sirvan para sacar conclusiones, pero desde luego apelan a nuestras intuiciones. $\mathrm{Y}$ aunque sea de un modo exploratorio, pueden servir incluso para ver en qué medida la subjetividad es importante para nuestro concepto de "persona".

\section{Algunos problemas de los experimentos mentales literarios}

Ya hemos visto que concebir los experimentos mentales como bombas de intuición abre una puerta a su utilización. No obstante, las críticas contra la validez de los experimentos mentales que hemos revisado todavía podrían afectar a las bombas de intuición. Sin duda dejar de considerar los experimentos mentales bajo el modelo de los experimentos científicos reales debe rebajar nuestras exigencias. Por ejemplo, que "persona" sea un término de sentido común no parece ser un obstáculo para que podamos explorar sus poco determinadas implicaciones mediante una situación ficticia que nos ayude precisamente a comprender mejor la estructura interna del concepto. Pero como Dennett señala, también las bombas de intuición pueden ser problemáticas. Siendo así, parece necesario hacer algunas precisiones sobre su construcción para justificar plenamente su uso. En concreto me gustaría prestar atención a dos aspectos: el modo en que una bomba de intuición puede producir confusiones debido a una simplificación excesiva; y el tipo de posibilidad relevante para la construcción de un mundo posible en el que se desarrolle una bomba de intuición.

El problema de la simplificación fue señalado por Dennett al mismo tiempo que acuñaba el término "bomba de intuición" para criticar el experimento mental de la habitación china de Searle. De acuerdo con Dennett, el experimento mental de Searle es una mala bomba de intuición, pues al simplificar en exceso la situación que pretende analizar nos lleva a la falsa conclusión que Searle pretende defender (Dennett, 2013, pp. 319-320). Como el lector sin duda recordará, el experimento mental de Searle consiste en un hombre que no habla chino encerrado en una habitación junto con un libro de normas sobre cómo manipular símbolos chinos. En esas circunstancias, según Searle, el hombre sería capaz de mantener una conversación en chino por escrito con alguien que se encontrase fuera de la habitación únicamente siguiendo las normas del libro. Sin embargo, el hombre de la habitación no comprendería chino. Siendo así, lo que el experimento pretende demostrar es que un ordenador que poseyese en su programación una serie de normas sobre cómo manipular símbolos chinos, tampoco sería capaz de comprender chino. Incluso aunque desde fuera pudiese parecer que sí (vid. Searle, 1980).

No obstante, tal y como Dennett señala, la presentación del experimento que hace Searle ignora algunos hechos que sesgan el resultado. Por ejemplo, no señala que siguiendo el manual de normas el hombre situado dentro de la habitación china necesitaría un tiempo enormemente largo para ofrecer una respuesta cualquiera a su interlocutor, pues tendría que hacer billones de cálculos manualmente. Si Searle señalase este hecho quizá nuestra respuesta intuitiva sería que la habitación china no sería capaz de "engañar" a nadie haciéndole creer que alguien en su interior habla chino. Por poner otro ejemplo, al introducir un hombre dentro de la habitación para hacer un trabajo mecánico Searle nos distrae del hecho de que quien en todo caso sería capaz de comprender chino no sería el hombre en sí, sino el sistema completo formado por el hombre junto con el libro de normas. Una neurona cualquiera per- 
teneciente al cerebro de un ciudadano chino tampoco comprende por sí misma el idioma. El conjunto de todas las neuronas de ese cerebro, junto al resto del cuerpo que posibilitan su trabajo, forman un sistema que es capaz de comprender chino, pero ninguna de sus partes tomadas por separado es capaz de hacerlo (Dennett, 2013, pp. 324-328).

La lección que podemos aprender del análisis que hace Dennett de la bomba de intuición de Searle es que no debemos apresurarnos a la hora de extraer conclusiones. El experimento de la habitación china no es por sí mismo "malo", como Dennett piensa. Por el contrario, la interpretación que hace Searle de él sí lo es. A pesar de que la bomba de intuición de Searle haya provocado que multitud de gente piense erróneamente que un ordenador nunca podrá comprender lo que hace, cabe preguntarse si explorar sobre dicha bomba de intuición, por engañosa que sea, no puede ayudarnos a descubrir el engaño y por tanto a comprender mejor qué aspectos del trasfondo influyen en el experimento pero están siendo ignorados en el mismo. Esta reflexión nos conecta de nuevo a la primera crítica señalada por Wilkes: la importancia de determinar el trasfondo en los experimentos mentales.

Una bomba de intuición no tiene por qué, en un principio, determinar el trasfondo del mismo modo en que debe hacerlo un experimento mental que siga el modelo de los experimentos científicos reales. Sin embargo, a la hora de interpretarlo y reflexionar sobre él, se debe tener en cuenta que la bomba puede estar ocultando aspectos relevantes para el problema. Es por lo tanto necesario que el filósofo interesado en el problema de la identidad personal que quiera utilizar bombas de intuición se detenga en explorar cómo distintos aspectos que rodean al experimento podrían tener un papel relevante para la interpretación que se pueda hacer del mismo. En este sentido la literatura presenta una ventaja evidente: aunque sea únicamente por su extensión, las obras literarias rara vez presentan una situación aislada y de forma esquemática tal y como sucede en los experimentos mentales tradicionales. Por el contrario, dedican mucho espacio a explorar la psicología de los personajes, sus relaciones y su entorno tanto antes como durante y después de una determinada situación. De este modo la literatura puede servir como un vasto repertorio de bombas de intuición desarrolladas a las que el filósofo puede recurrir para explorar los distintos factores que afectan al problema de la identidad personal.

Finalmente, en cuanto al tipo de posibilidad relevante para la construcción de mundos posibles en los que se desarrolle una bomba de intuición, parece necesario hacer una aclaración. Como decíamos anteriormente (véase §4), los mundos literarios no parecen estar regidos por la estricta posibilidad metafísica de la que hablaba Ricoeur. Lo que es más, pese a la afirmación menos demandante de Wilkes de que la literatura construye mundos lógicamente posibles, es un hecho bien conocido que las obras literarias a menudo presentan contradicciones lógicas, bien sea por un error involuntario o como parte integral del efecto estético buscado por el autor (vid. Ryan, 2013). Siendo así, y dado que tanto Wilkes como Ricoeur criticaban los experimentos mentales restringidos únicamente por el recurso a la posibilidad lógica por considerar ésta demasiado laxa, cabe preguntarse si el hecho de que la literatura ni siquiera esté realmente restringida por la posibilidad lógica no debería descartarla como recurso metodológico a la hora de utilizar bombas de intuición.

Sin embargo no creo que el hecho de que una obra literaria presente contradicciones lógicas deba considerarse como un impedimento para utilizarla como una bomba de intuición. Como ya hemos señalado, las bombas de intuición no son argumentos de los que 
tengamos que extraer conclusiones lógicas. Son una herramienta utilizada para explorar cómo se estructuran nuestros conceptos y comprender mejor todas las aristas de un determinado problema. En este sentido, incluso un experimento que contenga una contradicción lógica puede ayudarnos a comprender mejor una situación. De hecho, es posible que gran parte del problema de la identidad personal resida precisamente en el hecho de que nuestro concepto pre-teórico de "persona" tiene propiedades inconsistentes (Rovane, 1998, p. 11). En este sentido, la única restricción que debemos tener en cuenta, tanto a la hora de utilizar una bomba de intuición como a la de recurrir a las comparaciones con obras literarias, es la misma que acabamos de señalar al revisar las críticas de Dennett al experimento mental de Searle. No debemos apresurarnos a sacar conclusiones, sino detenernos a analizar con calma la situación para saber qué aspectos relevantes podríamos estar ignorando.

\section{Conclusiones}

Las críticas de Wilkes y Ricoeur contra el uso de los experimentos mentales van en sentidos contrarios. La primera propone su abandono por su excesivo parecido con las ficciones literarias. El segundo por el contrario justifica su rechazo en que tienen un carácter demasiado cientificista. Sin embargo, como hemos visto, en cierto sentido sus críticas se anulan mutuamente. Wilkes está en lo cierto al pensar que los experimentos mentales tienen un carácter literario. Sin embargo, esto por sí mismo no justifica su abandono. De hecho Ricoeur piensa que sería más apropiado sustituir los experimentos mentales por ficciones plenamente literarias. Siendo así, las teorías narrativas de la identidad, al aceptar las comparaciones entre lo que somos y los personajes literarios se estarían comprometiendo a aceptar también la metodología de los experimentos mentales. Sin embargo lo deben hacer con un espíritu radicalmente distinto al de los experimentos mentales de inspiración científica. No deben ser utilizados como argumentos a favor de una postura concreta. Por el contrario deben utilizarse de forma exploratoria para mejorar nuestra comprensión de nuestras propias creencias e intuiciones acerca de lo que constituye nuestra identidad personal.

\section{Bibliografía}

Beck, S. (2006). These Bizarre Fictions: Thought Experiments, our Psychology and our Selves. Philosophical Papers, 35(1), 29-54.

Benito Vicente, J. O. (2003). El problema de la identidad personal en la filosofía analítica. Daimon, Revista de Filosofía, 28, 67-83.

Coleman, S. (2000). Thought Experiments and Personal Identity. Philosophical Studies, 98(1), 53-69.

Dennett, D. C. (1992). The Self as a Center of Narrative Gravity. In F. S. Kessel, P. M. Cole, \& D. L. Johnson (Eds.), Self and Consciousness: Multiple Perspectives (pp. 103-115). Hillsdale, NJ: Erlbaum.

Dennett, D. C. (2013). Intuitions Pumps and Other Tools for Thinking. New York: W. W. Norton \& Company.

Diamond, C. (2002). What if $\mathrm{x}$ isn't the number of sheep? Wittgenstein and ThoughtExperiments in Ethics. Philosophical Papers, 31(3), 227-250. 
Gendler, T. S. (1998). Exceptional Persons: On the Limits of Imaginary Cases. Journal of Consciousness Studies, 5(5-6), 592-610.

Gendler, T. S. (2002). Personal Identity and Thought Experiments. The Philosophical Quarterly, 52(206), 34-54.

Johnston, M. (1987). Human Beings. The Journal of Philosophy, 84(2), 59-83.

Lamarque, P. (2007). On the Distance between Literary Narratives and Real-Life Narratives. In D. D. Hutto (Ed.), Narrative and Understanding Persons (pp. 117-132). Cambridge: Cambridge University Press.

Locke, J. (1975). An Essay Concerning Human Understanding. Oxford: Oxford University Press.

MacIntyre, A. (2007). After Virtue: A Study in Moral Theory (Third Edition). Notre Dame, IN: University of Notre Dame Press.

Martin, R. (1998). Self-Concern: An Experiential Approach to What Matters in Survival. Cambridge: Cambridge University Press.

Muñoz-Corcuera, A. (2016). Persons, Characters and the Meaning of 'Narrative'. In R. Winkler (Ed.), Identity and Difference: Contemporary Debates on the Self (pp. 37-61). London: Palgrave Macmillan.

Parfit, D. (1984). Reasons and Persons. Oxford: Oxford University Press.

Quine, W. O. (1972). Review of Identity and Individuation, by Milton K. Munitz. The Journal of Philosophy, 69(16), 488-497.

Ricoeur, P. (1990). Soi-même comme un autre. París: Éditions du Seuil.

Ricoeur, P. (1996). Sí mismo como otro. Madrid: Siglo XXI.

Romerales, E. (1993). ¿Consiste en algo ser una y la misma persona? Daimon, Revista de Filosofía, 6, 97-117.

Rorty, R. (1980). Philosophy and the Mirror of Nature. Princeton, NJ: Princeton University Press.

Rovane, C. (1998). The Bounds of Agency. Princeton, NJ: Princeton University Press.

Ryan, M.-L. (2013). Impossible Worlds and Aesthetic Illusion. In W. Wolf, W. Bernhart, \& A. Mahler (Eds.), Immersion and Distance: Aesthetic Illusion in Literature and Other Media (pp. 129-146). Amsterdam: Rodopi.

Schechtman, M. (1996). The Constitution of Selves. Ithaca, NY: Cornell University Press.

Searle, J. R. (1980). Minds, Brains, and Programs. Behavioral and Brain Sciences, 3(3), 417-424.

Shoemaker, S. (1964). Self-Knowledge and Self-Identity. New York: Cornell University Press.

Sorensen, R. A. (1999). Thought Experiments. Oxford: Oxford University Press.

Stokes, P. (2013). Will it be me? Identity, concern and perspective. Canadian Journal of Philosophy, 43(2), 206-226.

Taylor, C. (2001). Sources of the Self: The Making of Modern Identity. Cambridge, Mass.: Harvard University Press.

van Inwagen, P. (1997). Materialism and the Psychological-Continuity Account of Personal Identity. Noûs, 31(Supplement: Philosophical Perspectives, 11, Mind, Causation and World), 305-319.

Vice, S. (2003). Literature and the Narrative Self. Philosophy, 78(303), 93-108. 
Wilkes, K. V. (1988). Real People: Personal Identity without Thought Experiments. Oxford: Clarendon Press.

Williams, B. (1957). Personal Identity and Individuation. Proceedings of the Aristotelian Society, 57, 229-252.

Williams, B. (1970). The Self and the Future. The Philosophical Review, 79(2), 161-180.

Zarnitsyn, A. (2013). Thought Experiments in Personal Identity: A Literary Model (PhD Thesis). University of Illinois at Chicago, Chicago. 